\title{
INTEGRAÇÃO DAS METODOLOGIAS DO PDP E PMBOK NA ÁREA DE GESTÃO DO DESIGN
}

The iconography of the playing cards

ALVES, Milena Carneiro

Universidade Federal do Maranhão

mcarneiroalves@gmail.com

AZEVEDO, Patrícia Silva

Universidade Federal do Maranhão

psazeved@hotmail.com

\section{Resumo}

O desenvolvimento de produtos esteve atrelado desde o início das civilizações à racionalidade humana em satisfazer as suas necessidades. Com a evolução industrial, conceitos, gerenciamentos e processos de produção de objetos foram se modificando, buscando sempre a rapidez, mas com baixa eficiência e impactos negativos. Atualmente o Designer compreende informações metodológicas de diferentes áreas, viabilizando a melhor interação estratégica de produção. Assim, busca-se neste trabalho o aprimoramento do processo de Gestão em Design, por meio da integração de seus conceitos, com as metodologias do Processo de Desenvolvimento de Produto (PDP) e do Guide to Project Management Body of Knowledge (PMBOK).

Palavras-chave: Gestão em Design, PMBOK, PDP, Produção de produtos.

\section{Abstract}

The development of products has been linked since the beginning of civilizations to the human rationality to meet the human needs. With industrial development, concepts, management process and production processes were changing, always looking for a faster production, but it with low efficiency and negative impacts. Currently the Designer includes methodological information from different areas, aiming for a better strategic interaction into production. This article searches for the improvement of management in design process, through the integration of its concepts, with the methodologies of the Product Development Process (PDP) and the Guide to the Project Management Body of Knowledge (PMBOK).

Key words: Design Management Process, PMBOK, Product Development Process, Product Production. 


\section{INTRODUC̣ÃO}

O desenvolvimento de novos produtos ou serviços é um desafio estratégico continuo para empresas que querem competir de forma diferente e eficiente nos vários ramos do mercado, que demandam ciclos de vida de produção mais curtos, assim como custos e níveis de falhas de projetos cada vez menores. Além de precisar respeitar os fatores ambientais, políticos, tecnológicos, econômicos e sociais.

Estudos feitos em 2014 pela APEX Brasil, Centro Brasil Design, Ministério do Desenvolvimento da Indústria e Comércio do Exterior, relatados no Diagnóstico sobre o Design no Brasil, apontam que é necessário uma maior preocupação com os desenvolvimentos de novos produtos. Empresas que aplicam metodologias projetuais possuem sucesso significantemente alto (BERGER, 2013), onde essas metodologias podem ter nomes e etapas diferentes, mas possuem o mesmo propósito que é atingir os objetivos do projeto respeitando os requisitos definidos no escopo e plano de gerenciamento, de forma eficaz.

Baseando-se nesses dados, é evidente a necessidade, dentro do campo do Design, de uma maior utilização das metodologias de desenvolvimento de novos produtos, buscando a realização de uma produção mais estratégica, qualitativa, quantitativa, especializada e satisfatória para todas as partes interessadas.

Visando esse aperfeiçoamento produtivo, a proposta do estudo realizado foi a integração e utilização das metodologias do Processo de Desenvolvimento do Produto (PDP) desenvolvido por Rozenfeld et al. (2006) em seu livro "Gestão de Desenvolvimento de Produtos: Uma referência para a melhoria do processo", do "Guide to Project Management Body of Knowledge", registrado como $\mathrm{PMBOK}{ }^{\circledR}$ pelo Project Management Institute (PMI), e na área de Gestão em Design, a proposta publicada por Mozota et al. (2011) em seu livro "Gestão do Design: Usando o Design para construir valor de marca e inovação corporativa", aproveitando sua dinamicidade e flexibilidade em Design, para explorar, comparar e mostrar que com o auxílio da literatura, a integração dessas metodologias possibilita o desenvolvimento de novos produtos mais satisfatório para todas as partes interessadas.

\subsection{Gestão em Design}

O processo de Design fornecido por Mozota et al. (2011) tem um carácter multidisciplinar que integra pesquisa de mercado, estratégia de marketing, engenharia, desenvolvimentos de novos produtos, planejamento de produção e políticas de comunicação corporativa. É um processo criativo e possui seis fases: Investigação, Pesquisa, Exploração, Desenvolvimento, Realização e Avaliação. 
A fase da Investigação concebe ideias para gerar produtos que atendam às necessidades do usuário por meio de uma observação criteriosa sobe a problemática apresentada, precedendo a fase da Pesquisa, que está voltada para a concepção do conceito e de um esboço do diagnóstico do projeto baseados na estratégia desenvolvida na etapa anterior, sendo coerente com os objetivos e requisitos do projeto.

A fase da Exploração examina as soluções do projeto de forma funcional, técnica e estética, para gerar um produto inovador que produza reações positivas nas partes interessadas na fase do Desenvolvimento, onde esta representa formalmente o projeto com protótipos que obedecem as restrições técnicas e os fatores estéticos e funcionais.

Na Fase da Realização diferentes departamentos colaboram com o teste do produto, com a criação de documentos de execução e de um plano que define materiais e tratamentos de superfície, para que na fase da Avaliação, que é acompanhada pelo Designer, seja realizados testes de controle técnico e avaliação das respostas do mercado a respeito dos produtos lançados.

\subsection{Processo de Desenvolvimento de Produtos - PDP}

Segundo Rozenfeld et al. (2006), o PDP é um processo-chave de empresas que almejam liderança tecnológica por meio da redução do tempo de produção e criação de produtos cada vez mais competitivos e de excelência. Para que isto aconteça, são consideradas as necessidades, restrições e estratégias competitivas do mercado.

O PDP (figura 1) descreve recursos, informações, fases, responsabilidades e um conjunto de atividades em uma sequência lógica, com o objetivo de produzir um bem ou serviço que possua valor para todas as partes interessadas. Suas atividades são influenciadas por todas as pessoas da empresa e possui três Macro Fases: Pré Desenvolvimento, Desenvolvimento e Pós- Desenvolvimento. 
Figura 1: Quadro do PDP.

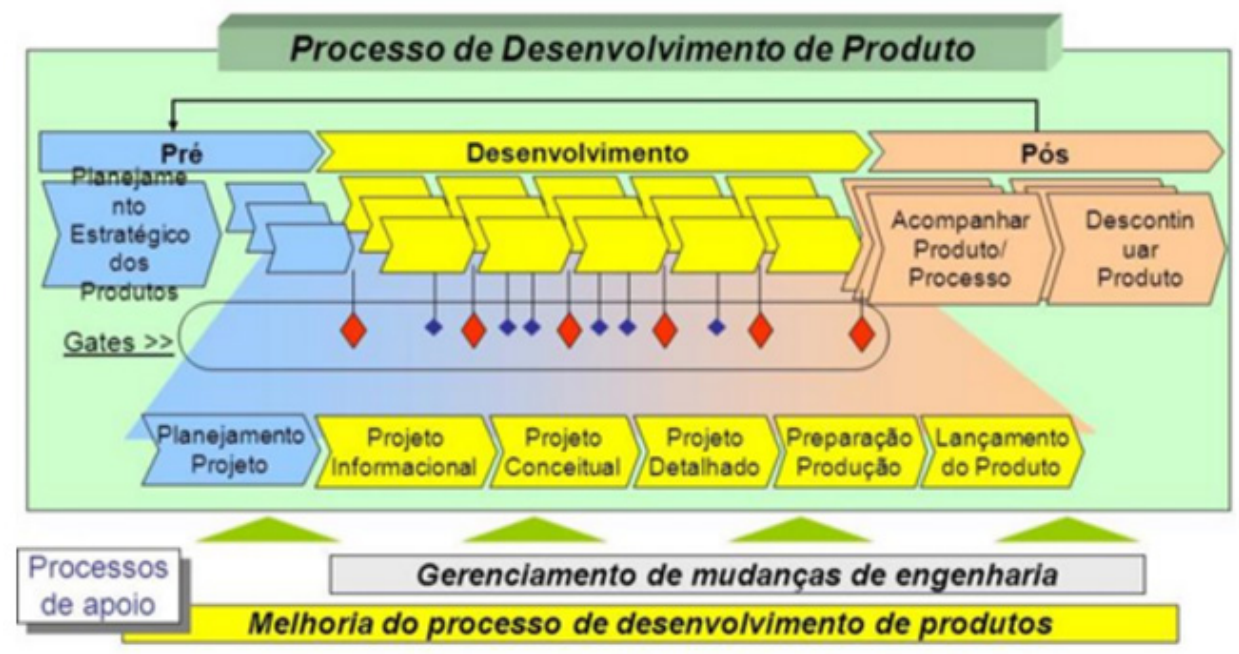

Fonte: Rozenfeld et.al (2006)

O Pré Desenvolvimento, abrange o Planejamento Estratégico dos Produtos e o Planejamento do Projeto, que define os objetivos, metas e requisitos que estarão listados no escopo do projeto. Considerando que Designers são capazes de integrar e coordenar os processos de desenvolvimento de produto (LORENZ, 1990, apud KANNO e SHIBATA, 2013), o cuidado em segregar as atividades que precisam ser feitas em etapas, logo no início do planejamento do projeto, ajuda a desenvolver produtos inovadores e mais valorizados no mercado, além de eliminar o desperdício e a desordem desde as fases iniciais do planejamento (MOZOTA et al., 2011)

O Desenvolvimento usa as informações concebidas na etapa anterior, enfatizando os aspectos tecnológicos dos produtos, suas características e as formas de produção. Suas subfases são: Projeto Informacional, Projeto Conceitual, Projeto Detalhado, Preparação para a Produção e Lançamento do Produto.

O Pós Desenvolvimento é uma etapa genérica que pode ser utilizada em qualquer empresa, sem precisar de grandes alterações e seu objetivo é acompanhar desempenho do produto no mercado e garantir que a sua retirada cause o menor impacto possível aos consumidores, empresa e meio ambiente. Possui duas subfases: Acompanhamento do Produto e do Processo e a Descontinuação do Produto no Mercado. A sua realização ajuda a empresa a aprender com os erros que aconteceram durante toda a execução e garante uma continuidade de conhecimentos contidos nos documentos de finalização do projeto de desenvolvimento de produtos (PDP).

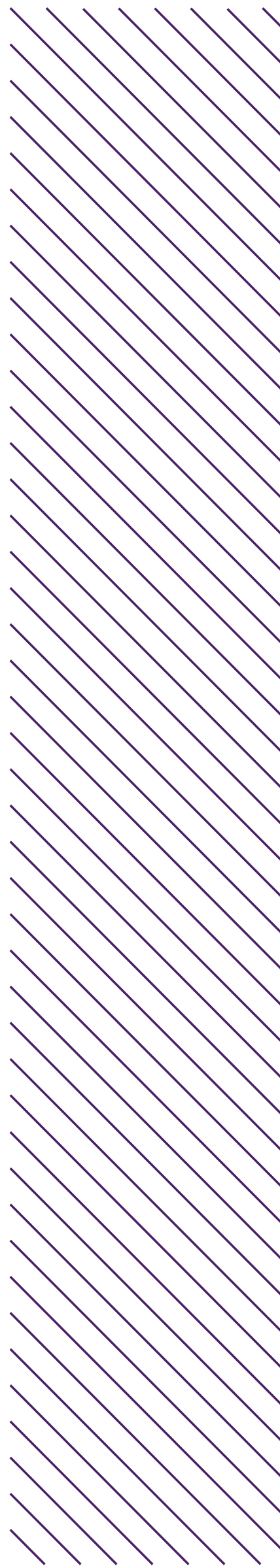




\subsection{Teorias do Guide to Project Management Body of Knowledge - PMBOK}

O Guia do PMBOK é um padrão que tem como objetivo a aplicação eficaz de habilidades, ferramentas e técnicas de conhecimentos no gerenciamento de projetos. E é reconhecido globalmente como um guia para a profissão de gerenciamento de projetos, podendo ser aplicado para diferentes tipos de produção.

Possui dez Áreas de Conhecimentos: Gerenciamento da Integração do Projeto, Gerenciamento do Escopo do Projeto, Gerenciamento do Tempo do Projeto, Gerenciamento dos Custos do Projeto, Gerenciamento da Qualidade do Projeto, Gerenciamento dos Recursos Humanos do Projeto, Gerenciamento das Comunicações do Projeto, Gerenciamento dos Riscos do Projeto, Gerenciamento das Aquisições do Projeto e Gerenciamento das Partes Interessadas do Projeto. E cinco Grupos de Processo: Iniciação, Planejamento; Execução; Monitoramento e controle; e Encerramento.

Os grupos de processos são guias para a aplicação de conhecimentos, habilidades e das áreas de conhecimentos do gerenciamento de projetos. $O$ Grupo de Processo de Iniciação ajuda a definir o escopo do projeto, os recursos financeiros, identifica as partes interessadas e os objetivos do projeto, sempre delineando estratégias e cursos de ação para uma conclusão com sucesso.

O Grupo de Processo de Execução coordena pessoas e recursos, gerencia as expectativas das partes interessadas e executa todas as atividades necessárias para concluir o trabalho definido pelo Plano de Gerenciamento. Enquanto o Grupo de Processo de Monitoramento e Controle acompanha, monitora, analisa, controla e organiza o progresso e o desempenho do projeto para que o Grupo de Processo de Encerramento execute e finalize todas as atividades propostas.

\section{METODOLOGIA}

Após a revisão da literatura, foi feita a comparação das metodologias do PMBOK (2013) e do PDP (2006) com as seis fases da Gestão do Design (2011) que buscou pontos em comum entre elas, para promover um desenvolvimento de produto mais qualitativo.

Com o objetivo de ajudar na visualização do quadro geral do resultado do estudo proposto, utilizou a teoria das cores (FARINA; PEREZ; BASTOS, 2006), 
para ajudar na identificação das simbologias através dos significados da sua essência, assim, foram escolhidas seis cores que se adequam com cada etapa dos processos estudados (figura 2).

Figura 2: Cores Utilizadas.

\begin{tabular}{|c|c|c|c|}
\hline CORES & Laranja & Azul & Amarelo \\
\hline CARACTERISTICAS & $\begin{array}{l}\text { - Equilibrio } \\
\text { - Criatividade } \\
\text { - Entusiasmo }\end{array}$ & $\begin{array}{l}\text { - Desenvolvimento } \\
\text { - Riqueza } \\
\text { - Fertilidade }\end{array}$ & $\begin{array}{l}\text { - Suavidade } \\
\text { - Sofisticaça } \\
\text { - Delicadeza }\end{array}$ \\
\hline FASES & $\begin{array}{l}\text { - Estrategica } \\
\text { - Informacional } \\
\text { - Organizacional }\end{array}$ & $\begin{array}{l}\text { - Pesquisa } \\
\text { - Aprimoramento } \\
\text { - Tecnologia }\end{array}$ & $\begin{array}{l}\text { - EXploratórias } \\
\text { - Criativas } \\
\text { - Solucionadoras }\end{array}$ \\
\hline CORES & & & \\
\hline CARACTERISTICAS & $\begin{array}{l}\text { - Harmónia } \\
\text { - Tecnologia } \\
\text { - Frescor }\end{array}$ & $\begin{array}{l}\text { - Concentraçà } \\
\text { - Disciplina } \\
\text { - Comunicaçao }\end{array}$ & $\begin{array}{l}\text { - Concentraçá } \\
\text { - Idealismo } \\
\text { - Velocidade } \\
\text { - Dinheiro }\end{array}$ \\
\hline FASES & $\begin{array}{l}\text { - Dezenvolvimento } \\
\text { - Execuça } \\
\text { - Coordenaça }\end{array}$ & $\begin{array}{l}\text { - Colaboraçăo } \\
\text { - Monitoramento } \\
\text { - Controle }\end{array}$ & $\begin{array}{l}\text { - Avaliaçao } \\
\text { - Testes } \\
\text { - Encerramento }\end{array}$ \\
\hline
\end{tabular}

Fonte: Autor, 2014

Em paralelo, foram desenvolvidos ícones que expressassem de forma gráfica cada etapa, fase e subfase das metodologias estudadas, o que permitiu que as comparações que foram realizadas fossem mais compreensíveis (figura 3).

Figura 3: Ícones desenvolvidos PMBOK (GUIA PMBOK, 2013).

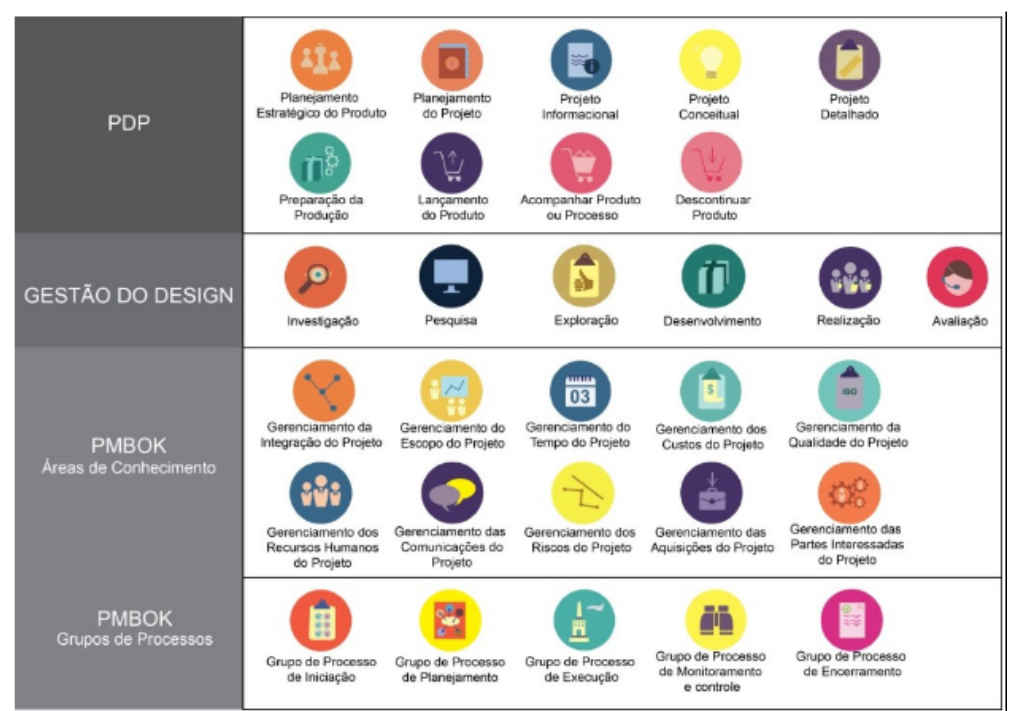

Fonte: Autor, 2014 


\section{RESULTADOS E DISCUSSÕES}

O estudo proposto provou que, a primeira fase da Gestão de Projetos, a Investigação, pode ser comparada com a macro fase do Pré Desenvolvimento do PDP, com as Áreas de Conhecimento de Gerenciamento da Integração, de Recursos Humanos e das Partes interessadas, e nos Grupos de Processo de Iniciação e Planejamento do padrão do PMBOK; onde todas apresentaram a característica de decidir, de forma mais eficaz, sobre o portfólio de produtos e de projetos, obtendo uma definição clara sobre os objetivos e considerando as estratégias tecnológicas do mercado.

A fase da Pesquisa (MOZOTA et al., 2011) foi comparada com a fase de Planejamento de Projeto e do Projeto Detalhado do PDP, assim como as Áreas de Conhecimento de Gerenciamento da Integração, dos Recursos Humanos, do Escopo e do Tempo, e os Grupos de Processo de Iniciação e Planejamento do padrão do PMBOK; onde todas almejam definir o Briefing, com o objetivo de obter o diagnóstico inicial de necessidades, custos e riscos visando à otimização das atividades que serão realizadas, garantindo assim, que a finalização do projeto possua um menor número de erros.

A terceira fase, a Exploração, foi comparada com o Projeto Conceitual e Detalhado do PDP, e com as Áreas de Conhecimento de Gerenciamento de Riscos, Comunicações e Escopo, e os Grupos de Processo de Planejamento, Execução, Monitoramento e Controle do Padrão do PMBOK. Todas compreendem o problema e concretiza o conceito, criando soluções baseadas em métodos de criatividade, desenvolvendo e finalizando as especificações do produto, acompanhando, monitorando, analisando e organizando o progresso e desempenho do projeto.

A etapa do Desenvolvimento (MOZOTA et al, 2011) foi comparada com a Preparação da Produção do Produto no PDP, as Áreas de Conhecimento de Gerenciamento de Custos e Qualidade, e o Grupo de Processo de Execução, onde há a produção do modelo em tamanho real para que possa ser testado, visando o cumprimento dos requisitos do projeto e garantindo que a empresa consiga produzir produtos no volume definido na Declaração do Escopo do Projeto, com as mesmas qualidades do protótipo.

A Realização (MOZOTA et al, 2011), foi comparada com a fase do Projeto Detalhado e do Lançamento do Produto no PDP, as Áreas de Conhecimento de Gerenciamento da Qualidade, das Comunicações e das Aquisições do projeto e os Grupo de Processo de Execução, Monitoramento e Controle do PMBOK, todas exigem a colaboração de diferentes equipes de projeto, dos fabricantes e dos fornecedores externos, para criar documentos de execução e um plano para definir os materiais utilizados, visando as especificações finais do projeto 
que atendem aos requisitos de riscos e qualidade.

A última fase, a Avaliação, foi comparada com a Macro Fase do Pós Desenvolvimento do PDP, assim como os Grupos de Processo de Encerramento do Padrão do PMBOK, onde todas garantem o acompanhamento do desempenho do produto na produção e no mercando, identificando as necessidades ou oportunidades de melhorias e garantindo que quando o produto for retirado, essa ação cause o menor impacto possível ao meio ambiente.

Assim, a comparação entre as três metodologias proposta no estudo, se torna visível na figura 4, na qual apresenta as principais características de cada uma e seus pontos em comum. A integração almejada é a junção das particularidades de cada uma, sempre aproveitando os melhores conceitos, objetivos, habilidades e técnicas a fim de buscar o aprimoramento do processo de Gestão em Design para que produtos ou serviços sejam desenvolvidos de forma mais prática e eficaz, atendendo sempre os requisitos de todas as partes interessadas.

Figura 4: Principais características e pontos em comum entre cada metodologia.

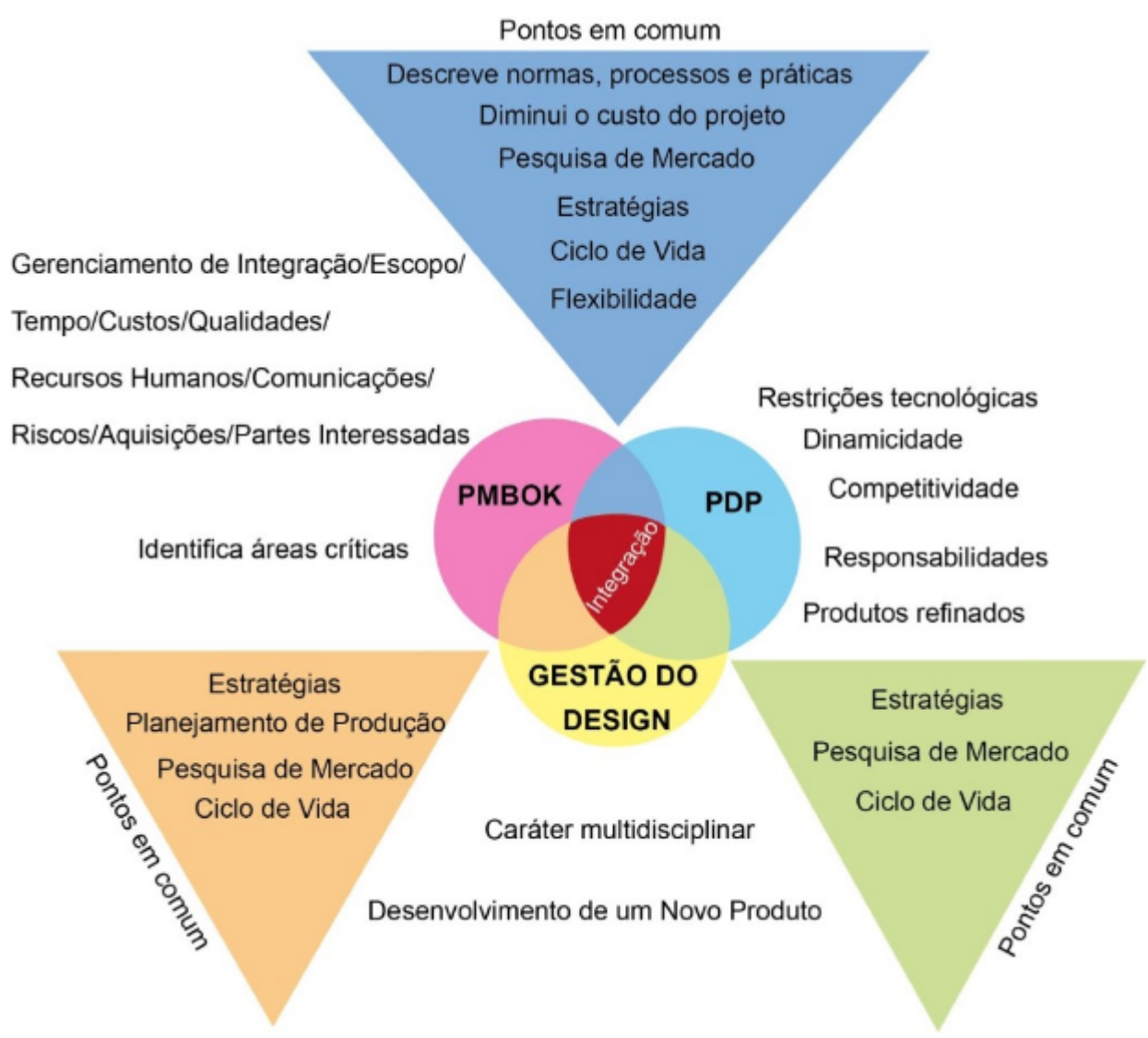

Fonte: Autor, 2014

Como resultado geral do estudo, criou-se um gráfico (figura 5) que contêm a junção das três metodologias (Gestão do Design, PDP e PMBOK) de acordo 
com a semelhança das suas características, que quando integradas, podem se adaptar para atender a diferentes tipos de projeto, tendo como auxilio todos os Grupos de Gerenciamentos e/ou fases (descritas no estudo), para produzir produtos únicos (GUIA PMBOK, 2013) e de sucesso (LIU, 2003).

Figura 5: Gráfico geral que envolve a integração da Gestão em Design (MOZOTA et al., 2011) do PDP (ROZENFELD et al., 2006) e do PMBOK (GUIA PMBOK, 2013).

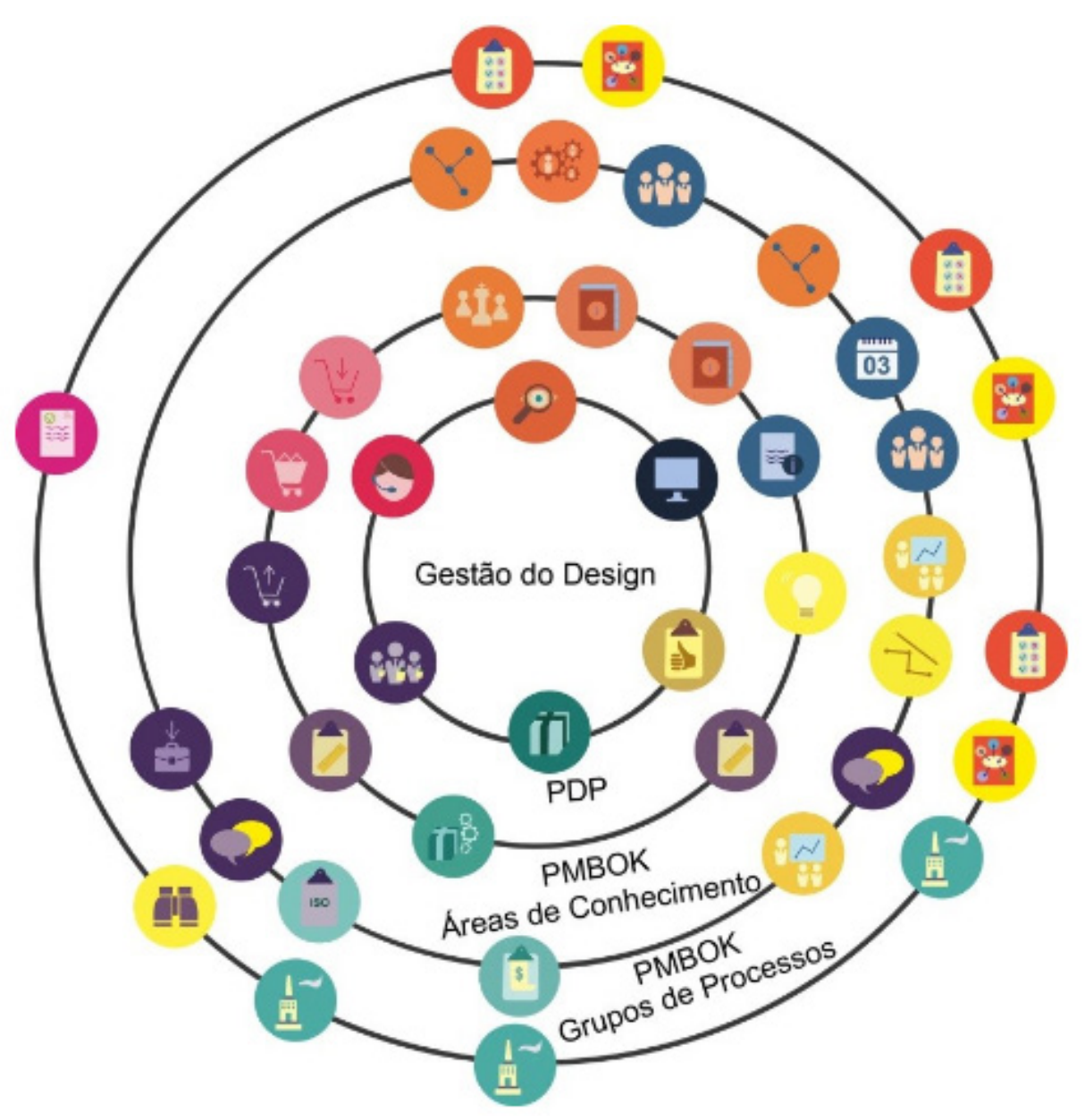

Fonte: Autor, 2014.

Dentro das relações estabelecidas, a cor laranja (utilizada para áreas estratégicas, informacionais e organizacionais) possui maior predominância. A Investigação (MOZOTA et al., 2011), possui relação direta com o Planejamento Estratégico e Planejamento do Projeto definidos por Rozenfeld et al. (2006), com o Gerenciamento das Partes Interessadas, da Integração e dos Recursos Humanos e com os Grupos de Processos de Iniciação e Planejamento (GUIA PMBOK, 2013). Pode-se notar que os ícones destes dois últimos foram confeccionados com as cores, laranja, amarelo e azul; esses dois Grupos de Processo são flexíveis e podem auxiliar as fases da Investigação, Pesquisa e 
Exploração (MOZOTA et al., 2011) por meio das suas ferramentas e técnicas.

A cor amarelo predominante na fase da Exploração (MOZOTA et al., 2011), também está diretamente ligada à fases executoras, criativas e solucionadoras, e por esse motivo, também se encontra presente, de forma sutil, nas fases do Desenvolvimento e Realização (MOZOTA et al., 2011). O Desenvolvimento encoraja interações e detalha restrições para solucionar os problemas e permite que a Realização crie os documentos de execução usando a colaboração de várias equipes que interagem entre as fases por meio do Gerenciamento da Qualidade e do Grupo de Processo de Execução (GUIA PMBOK, 2013).

A cor rosa possui a menor predominância no gráfico, a Avaliação (MOZOTA et al., 2011), está diretamente ligada apenas ao Acompanhamento do produto, Descontinuidade do produto (ROZENFELD et al., 2006) e ao Grupo de Processo de Encerramento (GUIA PMBOK, 2013).

\section{CONCLUSÃO}

O estudo e a comparação entre as metodologias da Gestão do Design (MOZOTA et al., 2011), do PDP (ROZENFELD et al., 2006) e do PMBOK (GUIA PMBOK, 2013), permitiu uma maior compreensão de cada processo de produção e áreas de conhecimento, se tornando evidente a semelhança entre as etapas dessas metodologias e a suas flexibilidades, que permitem que cada uma possa ser utilizada de acordo com o objetivo do projeto.

Os processos de desenvolvimento de produtos analisados nesta pesquisa, são preocupados com os requisitos definidos no escopo do projeto e integram as equipes de trabalho de forma especializada gerando uma comunicação mais eficiente e uma produção mais satisfatória. O monitoramento do desempenho do produto que foi lançado, a avaliação da satisfação do usuário e o registro das lições aprendidas, são etapas de metodologias diferentes, que podem ser integradas para gerar um referencial para projetos futuros, por meio da sua documentação.

Assim, esse estudo mostrou que a interação do processo de desenvolvimento de produtos (ROZENFELD et al., 2006) e o Padrão do PMBOK (GUIA PMBOK, 2013) na Gestão do Design (MOZOTA et al., 2011) promovem uma melhor eficiência no processo de criação, detalhamento, produção, monitoramento e lançamento de produtos de inovação e eficientes. 


\section{REFERÊNCIAS}

APEX BRASIL; CENTRO BRASIL DESIGN; MINISTERIO DO DESENVOLVIMENTO, INDUSTRIA E COMERCIO DO EXTERIOR. Diagnóstico sobre o design no Brasil. Brasilia. 2014

BERGER,R. Best Practices in new product development: Using effective methods to boost success. Brandenburgische Technische Universitat.2013

FARINA, M.; PEREZ, C.; BASTOS, D. Psicodinâmica das cores em comunicação. São Paulo. Editora Blucher. 2006

KANNO,Y.; SHIBATA, S. International Journal of Business and Management. Toronto. Volume 8. Canadian Center of Science and Education. 2013

LIU,B. Product Development Processes and their Importance to Organizacional Capabilities. Massachusetts Institute of Technology. 2003

MOZOTA, B. B.; KLOPSH, C.; COSTA, F. C. X. Gestão do design: usando o design para construir valor de marca e inovação corporativa. Porto Alegre: Bookman, 2011.

PROJECT MANAGEMENT INSTITUTE. Um Guia do Conhecimento em Gerenciamento de Projetos. 5 a Edição Traduzida. Pensilvânia. 2013.

ROZENFELD, H.; FORCELLINI, F. A.; AMARAL, D. C.; TOLEDO, J. C.; SILVA, S. L.;ALLIPRANDINI, D. H.; SCALICE, R. K. Gestão de desenvolvimento de produtos: uma referência para a melhoria do processo. São Paulo: Editora Saraiva, 2006 


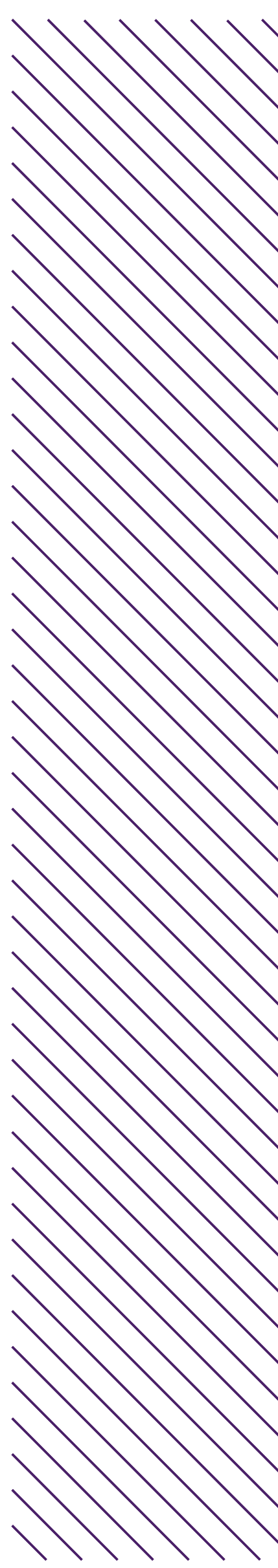

